\title{
Manifestações otoneurológicas tardia da malformação de Chiari I
}

\section{Late otoneurologic manifestations of Chiari I malformation}

\author{
Neide Fátima Cordeiro Diniz Oliveira', Renata Cristina Cordeiro Diniz Oliveira², Juliana Matosinhos de Paula². \\ 1) Mestrado em Ciências da Saúde. Diretora Clínica da Otomed e Preceptora do 11 e 12 período dos Acadêmicos da Faculdade Unifenas / BH. \\ 2) Acadêmica de Medicina da Faculdade de Medicina de Barbacena. \\ Instituição: Clínica OTOMED e Faculdade de Medicina de Barbacena \\ Belo Horizonte / MG - Brasil.
}

Endereço para correspondência: Renata Cristina Cordeiro Diniz Oliveira - Rua Ascanio Bulamarque, 199 - Bairro Mangabeiras - Belo Horizonte / MG - Brasil CEP: 30315-030 - Telefone: (+55 31) 3221-3630 - E-mail: dinizrenata@yahoo.com.br

Artigo recebido em 13 de Novembro de 2008. Artigo aprovado em 28 de Abril de 2011.

\section{RESUMO}

Introdução: A malformação de Chiari (MAC) pertence a um grupo de anomalias que envolvem as estruturas da junção crâniocerebelomedular. O tipo I (MAC I) é caracterizado pela descida das tonsilas cerebelares e da porção medial do lobo inferior do cerebelo pelo canal cervical. Na literatura, têm sido descritas manifestações motoras, sensoriais e autonômicas (5). É encontrada com mais frequência em mulheres. Sua prevalência é difícil de definir, pois existem muitos casos assintomáticos, o que torna escassa a informação epidemiológica (3).

Objetivo: O objetivo do trabalho foi relatar um caso de malformação de Chiari tipo I em uma paciente de 66 anos.

Relato de Caso: Os autores relatam um caso de malformação de Chiari tipo I, em paciente de 66 anos, com sintomas de zumbido, hipoacusia e cefaleia occipital.

Comentários Finais: A hipótese diagnóstica de Chiari tipo I deve ser embasada nas queixas do paciente, no exame clínico e de imagens, sendo a prevalência desta enfermidade de difícil definição, podendo haver diagnóstico em raras faixas etárias. Palavras-chave: vertigem, cefaleia occipital, zumbido, malformação de Chiari I.

\section{SUMMARY}

Introduction: Chiari malformation (CM) belongs to an anomaly group comprised of the structures in the cranial-cerebellarmedullary junctions. Type I (CM I) is characterized by the descent of the cerebellar tonsils and the medial portion of the lower cerebellar lobe through the cervical spinal canal. In literature, motor, sensorial and autonomous manifestations have been described. It is mostly found in women. Its prevalence is hard to determine, since there are many asymptomatic cases, hence, making the epidemiologic information scarce.

Objective: The objective of this work was to report a Chiari I malformation case in a 66-year-old female patient.

Case report: Authors report a Chiari I malformation case in a 66-year-old female patient, showing tinnitus, hearing loss and occipital headache symptoms.

Final Comments: Chiari I's diagnostic hypothesis must be based on the patient's complaints, clinical and image examinations, and since the prevalence of this disease is difficult to determine, there can be diagnoses in rare age groups.

Keywords: vertigo, occipital headache, tinnitus, Chiari I malformation. 


\section{INTRODUÇÃO}

A malformação de Chiari (MAC) pertence a um grupo de anomalias que envolvem as estruturas da junção crânio-cerebelo-medular. Foi descrita pela primeira vez por Cleland em 1883 (1) e mais tarde, por Chiari em 1891 (2) o qual reconheceu inicialmente três tipos: I, II, III e posteriormente o tipo IV. Os tipos II, III e IV geralmente são identificados antes ou ao nascimento e podem ser letais (2).

O tipo I (MAC I) é caracterizado pela descida das tonsilas cerebelares e da porção medial do lobo inferior do cerebelo pelo canal cervical, abaixo do plano do forame magno. No tipo II se observa o deslocamento das tonsilas cerebelares, vermis cerebelares e uma deformação de parte do quarto ventrículo e da medula oblonga em direção ao canal cervical. Os tipos III e IV envolvem grosseiras malformações craniais, com herniação de parte do cérebro e cerebelo (4).

Os sintomas iniciais do tipo I podem ocorrer na infância, mas na maioria dos casos os sintomas aparecem entre 30 - 50 anos de idade. É encontrada com mais frequência em mulheres, acompanhadas de manifestações motoras, sensoriais e autonômicas como cefaleia occipital, atrofia muscular e parestesia das extremidades superiores, que se agravam pela flexão, extensão cervical ou tosse (5). Sua prevalência é difícil de definir pois existem muitos casos assintomáticos, o que torna escassa a informação epidemiológica (3)

Desequilíbrio ou ataxia tem sido encontrado em 17 a 43\% dos pacientes (6). Mesmo sendo raros, vertigem e nistagmo têm sido descritos como sintomas de apresentação primaria do MACI (7). A literatura otolaringológica contém poucos relatos descrevendo o modelo de perda auditiva encontrada na MACI. Muitos casos consistem de revisões neurocirúrgicas de pacientes com significantes disfunções neurológicas (8).

No diagnóstico, além de uma anamnese detalhada e do exame físico, esta indicada a avaliação audiológica, provas vestibulares, além da ressonância magnética contrastada com gadolínio (7).

O tratamento cirúrgico é considerado para os pacientes que apresentam sintomas progressivos e debilitantes, como aumento da pressão intracraniana ou neuropatia autonômica (7).

Os autores relatam um caso com sintomas de zumbido, hipoacusia e cefaleia occipital, devido à malformação de Chiari tipo I.

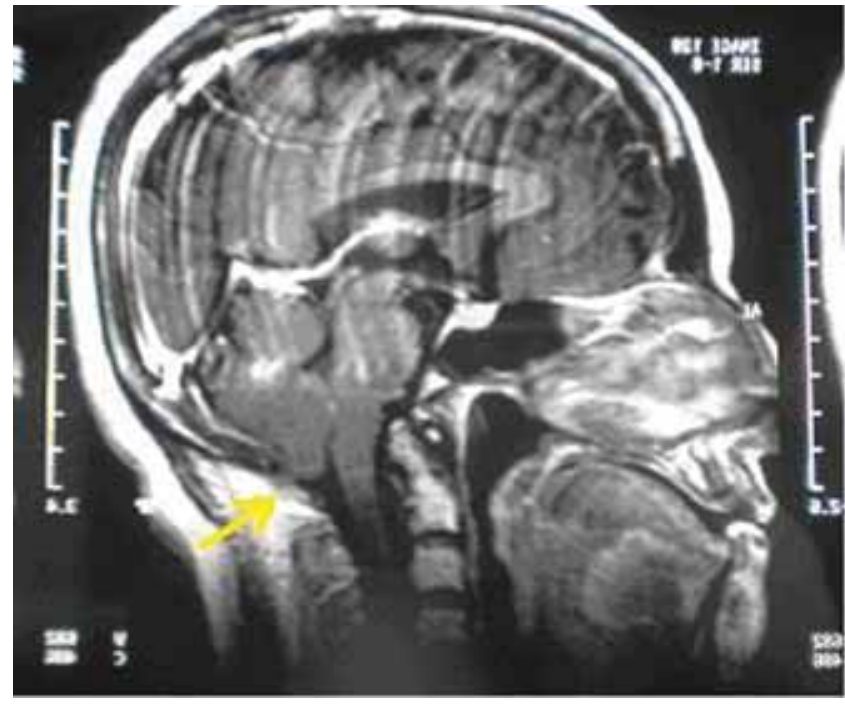

Figura 1. RNM de encéfalo mostrando MAC-I.

\section{Relato de CAso}

Paciente do gênero feminino, 66 anos, natural de Belo Horizonte / MG, veio a consulta com queixas de vertigem de início súbito, associado a náuseas e vômitos, com sensação de queda para trás e visão turva desde há dois dias. Referiu também cefaleia occipital, parestesia de membros superiores e plenitude auricular bilateral sem melhora após uso de analgésicos de dipirona e paracetamol. A otoscopia, rinoscopia anterior e oroscopia encontravamse sem alterações. Devido a intensidade dos sintomas a paciente foi encaminhada para internação para soroterapia e exames complementares.

Realizou-se investigação para descartar doença sistêmica e a paciente apresentou funçao renal e hepática normais, assim como glicemia, hemograma, provas de função tireoideana, cálcio, VDRL, vitamina B12 e acido fólico.

A tomografia computadorizada do segmento encefálico mostrou leve redução volumétrica do encéfalo e a ressonância nuclear magnética (RNM) evidenciou pequena projeção das amigdalas cerebelares pelo forame magno configurando MAC I (Figuras 1 e 2).

A avaliação neurológica confirmou o diagnóstico de MAC I e manteve tratamento conservador, devido a idade da paciente e da evolucão favorável do caso, com medicação sintomática e reabilitação vestibular.

Após alta hospitalar a avaliação oftalmológica incluindo o campo visual computadorizado mostrou-se dentro dos limites normais bilateralmente. 


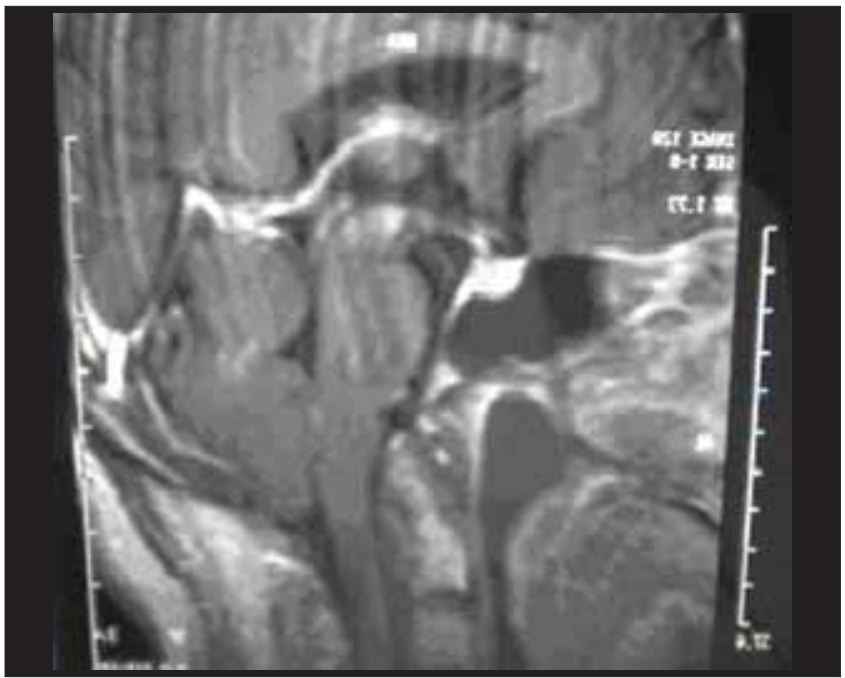

Figura 2. RNM de encéfalo mostrando MAC-I.

No décimo quinto dia de evolução, a audiometria tonal mostrou limiares tonais aereo-osseo variando de 25 dB à 60 dB (250 Hz a 8000Hz), com discriminação vocal de monossílabos de $96 \%$ em ambas orelhas e SRT de 35 dBNA. A imitanciometria encontrou curva tipo A com presença de reflexo estapediano contralateral. $\mathrm{Na}$ vectoeletronistagmografia não foram observados nistagmos espontâneos de olhos abertos e fechados e nem de posição. O Rastreio Pendular foi tipo III, o nistagmo optocinetico simétrico e normorreflexia vestibular à prova calórica.

\section{DISCUSSÃO}

Muitas teorias têm sido aceitas para explicar o envolvimento do VIII nervo na MAC I. As explicações para o comprometimento da orelha interna se devem a compressão do núcleo do nervo coclear intracerebral e da isquemia cóclear e do núcleo vestibular, resultante da torção da porção inferior da artéria cerebelar ou de um de seus ramos(4). Os episódios de instabilidade, vertigem e plenitude aural nos remete a Síndrome de Meniere, mais a presença de algum sinal ou sintoma neurológico associado (no caso da paciente, parestesia), nos fez pensar em Chiari.

Desequilíbrio ou ataxia tem sido encontrado em 17 a 43\% dos pacientes (6). Mesmo sendo raros, vertigem e nistagmo tem sido descritos como sintomas de apresentação primária da MAC I (7). A literatura otolaringológica contém poucos relatos descrevendo o modelo de perda auditiva encontrada na MAC I. Muitos casos consistem de revisões neurocirúrgicas de pacientes com significantes disfunções neurológicas (8).
No diagnóstico, além de anamnese detalhada e exame físico esta indicada a avaliação audiológica, provas vestibulares, alem da ressonância magnética contrastada com gadolínio. O tratamento cirúrgico é considerado para os pacientes que apresentam sintomas progressivos e debilitantes, como aumento da pressão intracraniana ou neuropatia autonômica.

A RNM é exame complementar importante de diagnóstico, em caso de suspeita de MAC I, devido às várias similaridades clínicas de diversas afecções.

A hipótese diagnóstica de Chiari tipo I deve ser embasada nas queixas, no exame clínico e de imagens.

\section{COMENTÁRIOS FINAIS}

A apresentação desse caso clínico se deve ao fato de se ter um diagnóstico de uma entidade rara na faixa etária da paciente.

\section{REFERÊNCIAS BIBLIOGRÁFICAS}

1. Cleland J. Contribution to the study of spina bífida, encefalocele, and anencephalus. Anat Physiol. 1883, 17:257-292.

2. Chiari H. Uber Veranderungen des Kleinhirns infolgevon Hydrocephalie des Grosshirns. Dtsch Med Wschr. 1891, 17:1172-1175.

3. Fonseca S. et al. Anestesia para Cesariana em doente com Malformacao Arnold Chiari tipo I e Seringomielia. Rev SPA. 2006, 16(2):24-28

4. Gonçalves da Silva JA, et al. Malformaçoes occipitocervicais, impressao basilar, malformacao de Chiari, seringomielia, platibasia. Recife: Editora Universitária; 2003, 169-300.

5. Sperling NM, Franco RA, Milhorat T and J R. Otologic Manifestation of chiari I malformation. The American Journal of Otology. 1994, 15:0 634-638.

6. Chiari H. Ueber Veranderunger des Kleinhirns infogle von Hydrocephalie des Grosshirns. Dtsch Med Wochenschr. 1891, 17:1172-5.

7. Albers FW, Ingels KJ. Otoneurological manifestation in Chiari-I malformation. J Laryngol Otol. 1993, 107(5):441-3.

8. Rydell RE, Pulec JL. Arnold Chiari malformation. Arch Otolaryngol. 1971, 94:8-12. 